\title{
A service process model for outsourcing human resources management decision support system: based on dynamic requirements perspectives
}

\author{
Jingyu Zhao \\ School of Management, Xiamen University, Xiamen Fujian, 361005, China \\ zjyplus@gmail.com
}

\begin{abstract}
Keywords: Decision Support System, Service Process Modeling, Dynamic Requirements, Modeling Tool
\end{abstract}

Abstract. Many companies changed their human resource management system into outsourcing human resources model. Outsourcing human resources management decision support system help these managers make their HR strategies. Compared to the traditional decision support system, outsourcing human resources management decision support system required high efficiency response and need to revise service process immediately. To acknowledge this research insufficient, this article firstly discussed the Composition of outsourcing human resources management decision support system. Then designed a service model for decision support system, which based on dynamic requirements perspectives. Finally, discussed the adaptability and reliability of the service process model and the whole decision support system.

\section{Introduction}

Based on the transformation of modern business model, more and more companies design their organization by the form of work teams. Thus outsourcing human resources management become more and more important for these companies. A well-designed outsourcing human resources management decision support system is a convenient tool for human resource managers to promote their work efficiency. For that outsourcing human resources management decision support system, there are two concerns. Firstly, the Composition of outsourcing human resources management decision support system, especially the databases that system required. Secondly, service process model for the outsourcing human resources management decision support system. Only a high efficient service process model could meet the dynamic service requirements from customers under the background of outsourcing human resources business model.

Outsourcing human resources management decision support system is different from the traditional human resource management system. These different reflect in the field of employee's career information and demographic information management, social network relationship management, mass information case analysis management, and so on. The whole system not only need to call and analysis mass information of a special employee for a customer to make decision, but also need to analysis those employees as a team together. That put forward higher requirements for outsourcing human resources management decision support system. And this article analysis the kind of database that is required in outsourcing human resources management decision support system.

The Traditional service process model focus on personalized requirements and build a service process model to meet customer's requirements. However, when customer revises their requirements or proposed some new requirement, the initial model will be dropped and then the system builds a new service model. That way of service process design makes the results of resource waste and management inefficiency. To solve this problem, this article proposed a service process model for customer's dynamic requirements. This model firstly divides customer's personalized requirements into three dimensions: functional, efficiency, and valuable. Furthermore, in order to meet the dynamic customized modeling requirements by using minimal modeling tools, this research define the modeling standard and service strategy design process. 


\section{Composition of outsourcing human resources management decision support system}

Outsourcing human resources management decision support system like the other decision support system. It including some necessary parts of a decision support system, including man-machine interface, DBMS, and database. Especially, the outsourcing human resources management decision support system required some special database to support the whole system. Also service provider need to maintain these database and updating service process models. This research designed the whole system as Fig. 1 displayed. And the following explain each part's function.

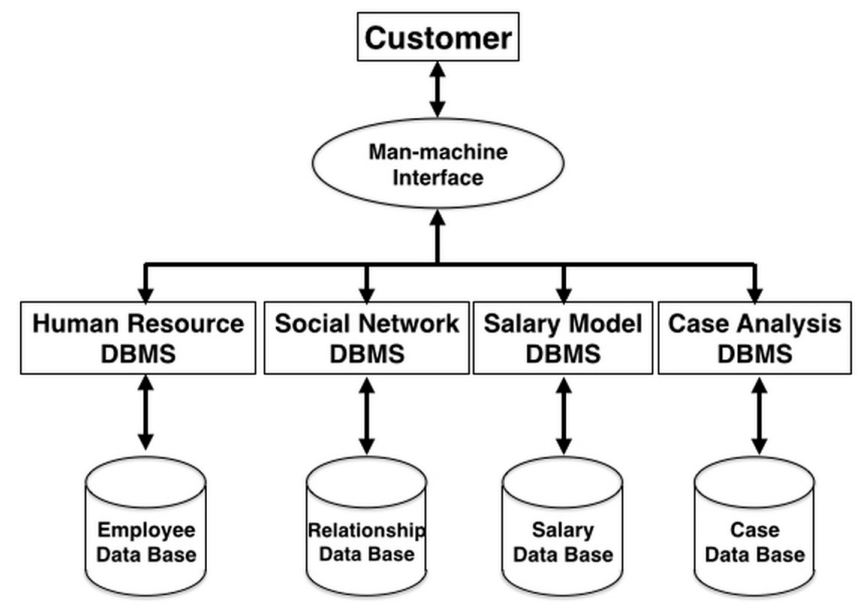

Fig. 1 Composition of outsourcing human resources management decision support system

Man-machine Interface. Man-machine interface is the basic software design for the whole system. Both customer and outsourcing human resources provider use that to make business decision and manage the system.

Human Resource DBMS. This database manages employee's demographic information. For example, age, gender, education level, tenure. The information of each employee who could be send to the new team work or have already been assigned to a project.

Social Network DBMS. This database manages employee's social relationship information. The relationships among those staff have a significant influence to the climate of project team. So the decision support needs to provide the information of relationships among those employees.

Salary Model DBMS. This database manages employee's salary information. As the outsourcing human resources business provide employee in the form of project cycle. Thus employee's salaries need to calculate for each project.

Case Analysis DBMS. This database manages history information of each employee's performance. Each piece of data generate from their previous project performance. That database more likely a performance database, but the data comes from case analysis.

To sum up, outsourcing human resources management decision support system needs software interface and powerful DBMS to keep it running. But the core of this decision support system is service process design. The strategies and business advise need designed carefully and accurate. That is why the composition of outsourcing human resources management decision support system been designed in this form, as displayed in Fig. 1. To fulfill that demand, the following of this article will discuss the service process model and proposed a service process model for outsourcing human resources management decision support system based on dynamic requirements perspectives.

\section{A service model for decision support system based on dynamic requirements perspectives}

The dynamic service process model has been divided into three layers, configuration layer, revised layer, and customization layer. Three layers combined together to fit dynamic customized requirements. This design demonstrates in Fig. 2. 


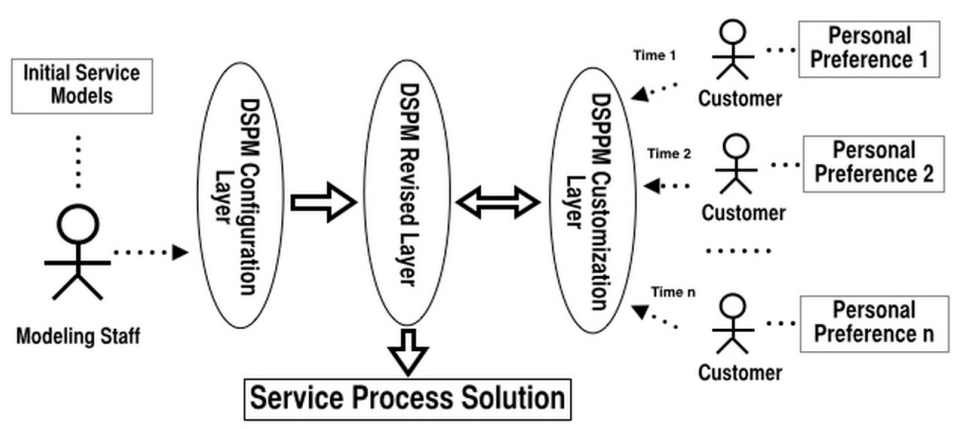

Fig. 2 Framework of service process model

As showed in Fig 2 these three layers combined and get connected by each other, also each part play a special roles itself. Firstly, configuration layer is build for outsourcing human resource provider staff. Service provider can set the basic service template and the relevant variable location, also manage and update the database of whole system. Secondly, customization layer is build for customer. Customer can set their personalized preference on the specific template that has been generated by configuration layer. Thirdly, revised layer is the bridge of those two layers mentioned above. This layer deals the dynamic requirement from customers, continuously generate the service process solution and transfer these feedbacks to the customer. So customer could revise their requirements and propose new requirements during the whole service process and new service process model deliver instantly. Based on analysis above, the whole service generate process could demonstrate by Fig. 3.

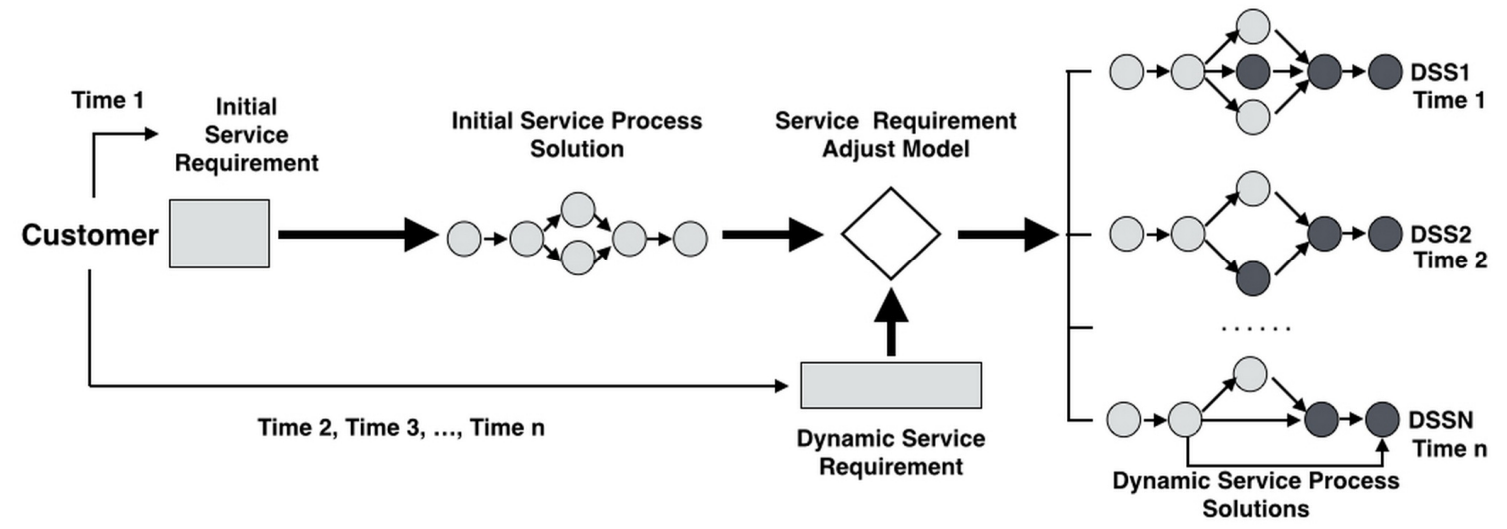

Fig. 3 Process of a service model for dynamic requirements

According to the design above, a modeling tool have been defined as following. Swimlane $=($ info,type $)$, in where info refers the customer's personal requirement, type refers the requirement type of task. Service assignment can be defined as Task $=\left(T_{-} I D, T_{-}\right.$Name, $T_{-}$Type $T_{-}$Choose $)$, in where $T_{-} I D$ refers to assignment ID; $T_{-}$Name refers to

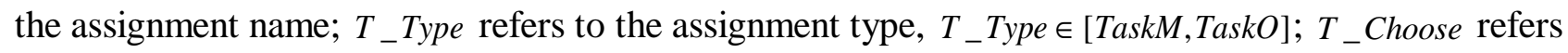
to customer' $\mathrm{s}$ choice, $T_{-}$Choose $\in[0,1]$. Also in a specific service process model task can be defined as Task $=($ info, Res, $B, C, E, C O N S)$, in where Res refers the resource wanted by a specific service process, $B$ refers to observable benefit of a specific assignment, $C$ refers to observable cost of a specific assignment, $E$ refers to latent benefit of a specific assignment, CONS refers to control conditions. The modeling tool defined variable point of a service process as $V P=($ Task,Task_Res,Task_Task,Task_QoS,Task_Service $)$, in where $Q o S$ refers quality of each assignment. Then define process connection line, $P C L=($ info, $S O, E O, C O N S)$, in where $S O, E O$ refers to the initial state of service and economic benefit. Service quality required $Q R=\left(Q o S_{-} I D, Q o S_{-}\right.$Name, $Q o s_{-}$Value $)$, in where ID, Name, Value refers the basic information of a specific service. Define task relationship links, $T_{-} T s=($ info, $S O, E O)$. To sum up, all the elements including the model must obey the attribute set. And then discussed the modeling rules. 
Configuration Layer. This research focused on configuration layer modeling rules. The modeling rules are set as follows. First, if VP_Position $=\{$ Task_Res or Task_QoS or B_CONB or C_CONC or $\left.E_{-} C O N E\right\}$, then staff can configure a lot of variable positions, process connector's start object corresponding to service assignment. Second, if VP_Position=Task, then Task=TaskO . Third, if $V P \_P o s i t i o n=T a s k \_T a s k$, let $T \_T S$ connect all the service assignments. Process connector object should be the beginning of a variable point. Fourth, if $V P \_P o s i t i o n=S$ Service_QoS, then set related service quality variable position to the customized value. Process connector object should be connecting to $Q R$ graphics primitive.

Customization Layer. Also focused on customization layer modeling rules, and the modeling rules are set as followings. First, all Task_ID must be the same for one service model. Second, about the value set of Service_QoS and Task_QoS. The value of $Q o S$ in Customization layer must be a subset of $Q o S$ in configuration layer.

Revised Layer. Revised layer need to deal with the dynamic service requirements. The modeling rules of this layer defined as followings. First, if Service_Task ${ }_{n} \neq$ Service_Task $_{0}$, then $V P \_$Position $=\left\{\right.$Task $\_$Res $\left._{n}, T a k_{-} Q_{o} S_{n}, B_{n}, C_{n}, E_{n}, C O N S_{n}\right\}$, in where footnote $\mathrm{n}$ refers to the new requirement from customers in time n. Second, set Service_Qo $S_{n}=F$ (Service_Qos, $n, C, C o n s$ ), in where F refers the Service Requirement Adjust Model as showed in Fig. 3. This function model compare the service process elements from new requirement with the initial service process elements, and finally select the most convenient parts to the final solution.

\section{Conclusion}

Outsourcing human resource become more and more popular in modern business model. The demand for high performance outsourcing HR decision support system increases a lot. To acknowledge this market trend this article proposed a system design plan and further more a service process model. Firstly, this research reported a way to build an outsourcing HR decision support system. Especially discussed the database that required supporting the solution design. Secondly, the service process model and modeling tool focused on dynamic requirement from customers. Three-layer design makes it efficient to revise initial service process instantly. To sum up, that makes the decision support system and service process-modeling tool have characteristic of popularity and applicability. Both customers and HR outsourcing providers can promote their operating efficiency through this system.

Though this research focus on the problems that need to solve, there are still something need further research. Firstly, about the adjust model. This research proposed a simple comparison equation. That equation could be more complicated and deliberated. Secondly, the service process model has several promotion ways, like classify customer's requirements. Thirdly, there are several database could add to the decision support system.

\section{References}

[1] Aguilar-Saven, Ruth Sara. "Business process modelling: Review and framework." International Journal of production economics. 90 (2004) 129-149.

[2] Jahid, Md Jamiul, Tim H. Huang, and Jianhua Ruan. "A personalized committee classification approach to improving prediction of breast cancer metastasis." Bioinformatics. 13(2014) 1858-1866.

[3] Wohed, Petia, et al. On the suitability of BPMN for business process modelling. Springer Berlin Heidelberg, 2006, pp.20-26

[4] Juric, Matjaz B., Benny Mathew, and Poornachandra G. Sarang. Business Process Execution Language for Web Services: An Architect and Developer's Guide to Orchestrating Web Services Using BPEL4WS. Packt Publishing Ltd, 2006, pp.55-65 